Disclosure of Interests: None declared

DOI: 10.1136/annrheumdis-2021-eular.3283

\section{POS0634 DO BIOLOGICS IMPROVE FATIGUE IN PATIENTS WITH RHEUMATOID ARTHRITIS?}

A. Fazaa ${ }^{1}$, H. Boussaa ${ }^{1}$, K. Ouenniche ${ }^{1}$, S. Miladi ${ }^{1}$, M. Sellami ${ }^{1}$, L. Souabni ${ }^{1}$, S. Kassab ${ }^{1}$, S. Chekili ${ }^{1}$, K. Ben Abdelghani ${ }^{1}$, A. Laatar ${ }^{1} .{ }^{1}$ Mongi Slim University Hospital, Rheumatology, Sidi Daoud, Tunisia

Background: Fatigue is a significant issue in rheumatoid arthritis (RA) with no accepted evidence-based management guidelines. Several studies suggested that biologic Disease Modifying Anti-Rheumatic Drugs (bDMARDs) have a direct role on fatigue in RA. Objectives: This study aimed to compare fatigue between patients treated with bDMARDs and conventional synthetic Disease Modifying Anti-Rheumatic Drugs (cs DMARDs)

Methods: We conducted a longitudinal study including patients with RA (ACR/ EULAR 2010). Patients with other acute or chronic diseases that may induce fatigue (such as cancer, infection or depression) were excluded. Demographic data and the following disease-related parameters were collected: pain Visual Analog Scale (VAS), Global Patient Assessment (GPA), tender joint count (TJC), swollen joint count (SJC), Erythrocyte Sedimentation Rate (ESR), C Protein Reactive (CRP), Disease Activity Score 28 (DAS28), Health Assessment Questionnaire (HAQ) and DMARDs used. Fatigue was assessed at baseline (T0), at 6 months (T6) and at 12months (T12) using the Functional Assessment of Chronic Illness Therapy - Fatigue (FACIT-F) which is a short 13-item questionnaire validated in RA. The score FACIT-F ranges between 0 and 52. Fatigue was considered mild if the FACIT-F score was $\geq 40$, moderate if $20 \leq F A C I T-F<40$ and severe if $0 \leq F A C I T-F<20$. A p value inferior to 0.05 was considered significant.

Results: We included 100 RA patients ( 84 women and 16 men) with a mean age of $49.5 \pm 10$ years old [18-65]. The mean disease duration was 87.3 months [1-360]. The mean pain VAS was $49 \mathrm{~cm}$ [0-100] and the mean GPA was $47.8 \mathrm{~cm}$ [0-100]. The mean TJC and SJC were 5.3 [0-36] and 1 [0-9] respectively. The mean levels of ESR and CRP were $38.1 \mathrm{~mm}$ [10-120] and $10.8 \mathrm{mg} / \mathrm{l}$ [2-61] respectively. The mean DAS28 ESR was 3.68 [1.90-8.33] and the mean HAQ score was 0.90 [0-2.75] Eighty-three percent of patients used csDMARDs: Methotrexate $(n=96)$, sulphasalazine $(n=28)$, leflunomide $(n=21)$, and hydroxychloroquine $(n=12)$. bDMARDs were prescribed in $17 \%$ of patients: Rituximab $(n=10)$, Infliximab $(n=9)$, and Etanercept $(n=5)$. At baseline, the mean FACIT-F score was 27.1 [0-51]. Moderate fatigue was noted in $57 \%$ of cases and severe fatigue in $26 \%$ of cases. Patients on csDMARDs had a lower FACIT-F score when compared to patients on bDMARDs (26.89 versus $28.41)$, but the difference was not statistically significant $(p=0.630)$

The mean FACIT-F score was 27.41 in bDMARDs patients versus 29.80 in csDMARDs patients $(p=0.497)$ at $T 6$, and 32.35 versus 33.65 respectively at $T 12(p=0.695)$.

The mean delta FACIT-F was 2.18 in bDMARDs patiens versus 2.73 in csDMARDs patients between T6 and T0 ( $p=0.815)$, and 3.94 versus 7.2 respectively between $\mathrm{T} 12$ and T0 $(\mathrm{p}=0.807)$.

When considering all patients, a significant positive correlation was noted between delta FACIT-F and delta DAS28 at T6 $(r=0.418, p<0.001)$ and at T12 $(r=0.338, p<0.001)$.

Conclusion: RA patients treated with bDMARDs didn't show significant improvement of fatigue in comparison with those treated with csDMARDs. Further studies are needed to determine if biologics improve fatigue, and whether the improvement results from a direct action on fatigue or indirectly through reduction in disease activity. Disclosure of Interests: None declared

DOI: 10.1136/annrheumdis-2021-eular.3351

\section{POS0635 1 COMPARING THE ULTRASONOGRAPHIC EVALUATION IN PATIENTS WITH JAPANESE RHEUMATOID ARTHRITIS BETWEEN BARICITINIB AND TNF ANTAGONIST THERAPY}

Y. Kanayama ${ }^{1}$, A. Nagata ${ }^{2}$, M. Shimotake ${ }^{2}$, F. Miyachi ${ }^{2}$, K. Fujita ${ }^{2}$, M. Koyama ${ }^{2}$, S. Uno ${ }^{2} .{ }^{1}$ Toyota Kosei Hospital, Orthopedic Surgery and Rheumatology, Toyota, Japan; ${ }^{2}$ Toyota Kosei Hospital, Clinical Technology, Toyota, Japan

Background: Baricitinib (BAR) and TNF antagonist are the important therapeutic agent for the treatment of rheumatoid arthritis. However there is still few studies of improvement of ultrasonographic findings in RA treated comparison with BAR and TNF.

Objectives: To evaluate the clinical efficacy of BAR and TNF therapy patients with rheumatoid arthritis (RA) using ultrasonography (US).

Methods: Participants comprised 16 and 45 Japanese RA patients who had recently received BAR and TNF. All patients with a diagnosis of RA according to the 2010 ACR/EULAR criteria. Patients underwent clinical and laboratory assessments every 4 weeks from baseline to 24 weeks, and US assessments at baseline, 4 , 12 and 24 weeks. Gray scale (GS) and power doppler (PD) signals were scored using a semi-quantitative scale from 0 to 3 at $26(0-78)$ synovial sites (22 joints) in the following joints: bilateral first to fifth metacarpopharangeal (MCP) joints (dorsal recess); first interphalangeal (IP) and second to fifth proximal interphalangeal (PIP) (dorsal recess) joints; and the wrists (dorsal radial, median and ulnar). We evaluated the improvement of GS and PD score from baseline to week 24.

Results: In the patients receiving BAR $(n=16)$ and TNF $(n=45)$, the mean age was 55.9 vs 54.6 years old $(p=0.682)$, disease duration was 10.2 vs 6.1 years $(p=0.094)$ the rate of MTX use was $75 \%$ vs $89 \%(p=346)$, the mean MTX dose was 9.3 vs $10.2 \mathrm{mg} / \mathrm{w}(\mathrm{p}=0.443)$, the rate of ACPA positive was $94 \%$ vs $82 \%(p=0.476)$, DAS28ESR was 4.25 vs 4.61 ( $p=0.289)$, CDAl was 15.8 vs $18.5(p=0.210)$, GS score was 21.6 vs $16.3(p=0.436)$ and PD score was 15.0 vs $9.5(p=0.260)$. The degree of improvement respective changes in GS and PD score after 4, 12 and 24 weeks were as follows: GS: -7.2 vs -3.7 ( $p=0.268)$ and PD: -7.6 vs $-2.3(p=0.158)$ after 4 weeks, GS: -10.9 vs $-5.0(p=0.161)$ and PD: -9.2 vs $-3.8(p=0.049)$ after 12 weeks, GS: -12.9 vs $-6.1(p=0.485)$ and PD: -11.3 vs $-5.7(p=0.062)$ after 24 weeks between BAR and TNF (Fig.1, 2). Next, The improvement rate of respective changes in GS and PD score after 4,12 and 24 weeks were as follows: GS: $-23.8 \%$ vs $-11.6 \%(p=0.580)$ and PD: $-30.3 \%$ vs $-16.5 \%(p=0.343)$ after 4 weeks, GS: $-39.6 \%$ vs $-15.6 \%(p=0.129)$ and PD: $-47.1 \%$ vs $-30.8 \%(p=0.210)$ after 12 weeks, GS: $-52.2 \%$ vs $-22.2 \%(p=0.248)$ and PD: $-77.1 \%$ vs $-50.1 \%(p=0.048)$ after 24 weeks between BAR and TNF.

Conclusion: The present study provides evidence supporting both the BAR and TNF therapy showed improvement effect over time, but in a comparison between BAR and TNF, the PD score of BAR showed a siginificant improvement effect compared to TNF at 12 and 24 weeks. It was suggeted that BAR may improve inflammatory synovitis earlier compared to TNF

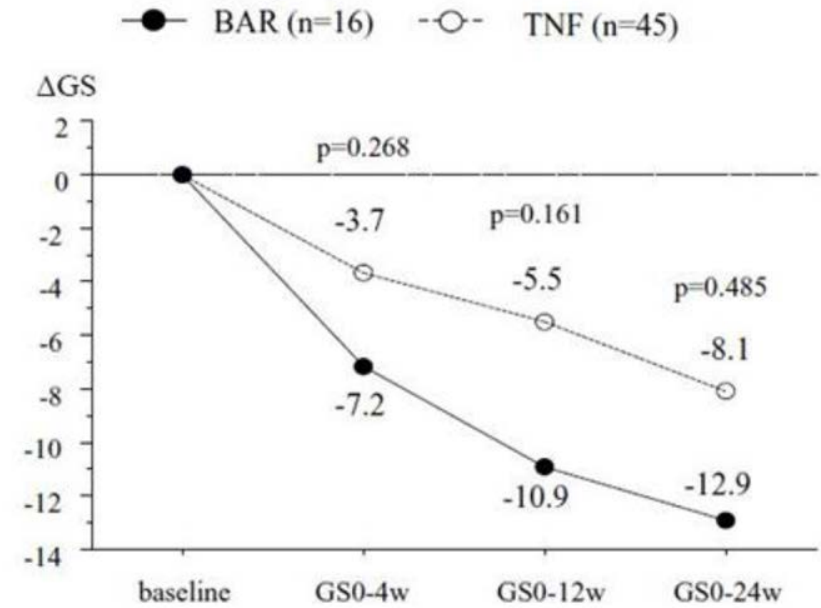

Figure 1: The degree of improvement respective changesin GS score from baseline to Week 24 between BAR and TNF patients

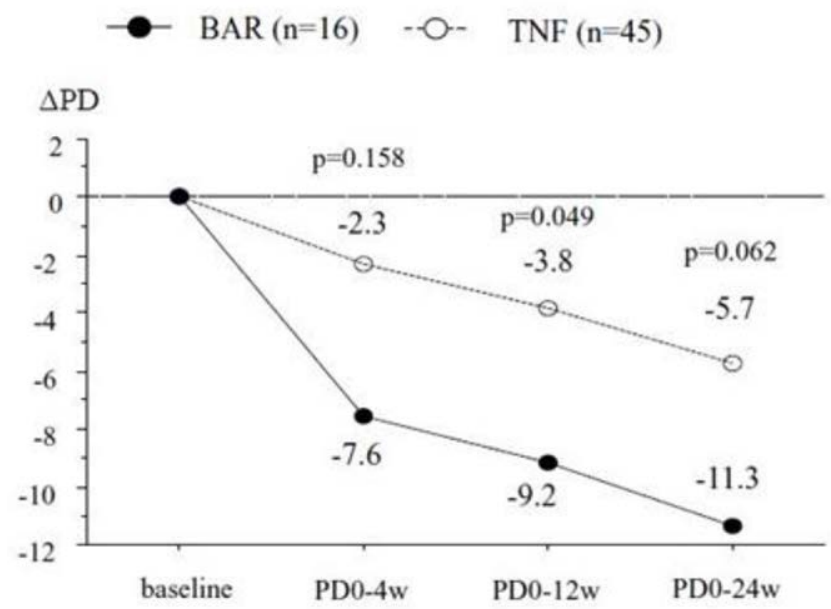

Figure 2 : The degree of improvement respective changesin PD score from baseline to Week 24 between BAR and TNF patients

Disclosure of Interests: None declared

DOI: 10.1136/annrheumdis-2021-eular.3483 